\title{
Synthesis of Molecular Oxygen via Irradiation of Ice Grains in the Protosolar Nebula
}

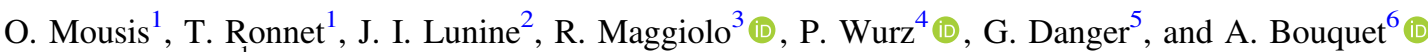 \\ Aix Marseille Univ, CNRS, CNES, LAM, Marseille, France; olivier.mousis@lam.fr \\ ${ }^{2}$ Department of Astronomy, Cornell University, Ithaca, NY 14853, USA \\ ${ }^{3}$ Royal Belgian Institute for Space Aeronomy, BIRA-IASB, Ringlaan 3, B-1180 Brussels, Belgium \\ ${ }^{4}$ Physikalisches Institut, University of Bern, Sidlerstrasse 5, CH-3012 Bern, Switzerland \\ 5 Aix-Marseille Université, PIIM UMR-CNRS 7345, F-13397 Marseille, France \\ ${ }^{6}$ Department of Space Research, Southwest Research Institute, 6220 Culebra Rd., San Antonio, TX 78228, USA \\ Received 2017 December 18; revised 2018 March 9; accepted 2018 March 12; published 2018 May 4
}

\begin{abstract}
Molecular oxygen has been detected in the coma of comet 67P/Churyumov-Gerasimenko with a mean abundance of $3.80 \pm 0.85 \%$ by the ROSINA mass spectrometer on board the Rosetta spacecraft. To account for the presence of this species in comet $67 \mathrm{P} /$ Churyumov-Gerasimenko, it has been shown that the radiolysis of ice grain precursors of comets is a viable mechanism in low-density environments, such as molecular clouds. Here, we investigate the alternative possibility that the icy grains present in the midplane of the protosolar nebula were irradiated during their vertical transport between the midplane and the upper layers over a large number of cycles, as a result of turbulent mixing. Consequently, these grains spent a non-negligible fraction of their lifetime in the disk's upper regions, where the irradiation by cosmic rays was strong. To do so, we used a coupled disk-transportirradiation model to calculate the time evolution of the molecular oxygen abundance radiolytically produced in ice grains. Our computations show that, even if a significant fraction of the icy particles has followed a back and forth cycle toward the upper layers of the disk over tens of millions of years, a timespan far exceeding the formation timescale of comet 67P/Churyumov-Gerasimenko, the amount of produced molecular oxygen is at least two orders of magnitude lower than the Rosetta observations. We conclude that the most likely scenario remains the formation of molecular oxygen in low-density environments, such as the presolar cloud, prior to the genesis of the protosolar nebula.
\end{abstract}

Key words: astrobiology - comets: general - comets: individual (67P/Churyumov-Gerasimenko) - methods: numerical - solid state: volatile

\section{Introduction}

Molecular oxygen $\left(\mathrm{O}_{2}\right)$ has been detected in the coma of comet $67 \mathrm{P} /$ Churyumov-Gerasimenko $(67 \mathrm{P} / \mathrm{C}-\mathrm{G})$ with abundances in the 1\%-10\% range and a mean value of $3.80 \pm$ $0.85 \%$ by the Rosetta Orbiter Spectrometer for Ion and Neutral Analysis-Double Focusing Mass Spectrometer (ROSINA) instrument on board the Rosetta spacecraft (Bieler et al. 2015). Moreover, the production rate of $\mathrm{O}_{2}$ has been found to be remarkably correlated with that of $\mathrm{H}_{2} \mathrm{O}$ in $67 \mathrm{P} / \mathrm{C}-\mathrm{G}$ 's coma, suggesting that both molecules come from the same icy phase (Bieler et al. 2015). A subsequent reanalysis of the Giotto mass spectrometry data shows that $\mathrm{O}_{2}$ was also present in the coma of comet $1 \mathrm{P} /$ Halley with an abundance of $3.7 \pm 1.7 \%$ with respect to water at the time of its encounter with the ESA spacecraft, suggesting that this species could be a common parent species in comets (Rubin et al. 2015).

To account for the $\mathrm{O}_{2}$ abundance and the correlation of its production rate with that of $\mathrm{H}_{2} \mathrm{O}$ in $67 \mathrm{P} / \mathrm{C}-\mathrm{G}$, it has been shown that the radiolysis of ice grains in low-density environments, such as molecular clouds, is a mechanism capable of producing large amounts of $\mathrm{O}_{2}$ from solid $\mathrm{H}_{2} \mathrm{O}$ (Mousis et al. 2016). Meanwhile, it was also found that the radiolysis of icy grains in higher density environments, such as the midplane of protoplanetary disks, is not efficient enough to create amounts of $\mathrm{O}_{2}$ comparable with those observed in $67 \mathrm{P} / \mathrm{C}-\mathrm{G}$ and $1 \mathrm{P} /$ Halley in timescales shorter than the lifetime of the protosolar nebula, even in the case of a strong cosmic-ray flux (CRF) engendered by the presence of a nearby supernova
(Mousis et al. 2016). It was then concluded that, if $\mathrm{O}_{2}$ is an irradiation product of ice, this molecule was probably formed in the interstellar medium, prior to the formation of the PSN.

Here, we consider the alternative possibility that the icy grains present in the midplane of the PSN were irradiated during their transport toward its upper layers (see the illustration in Figure 1). This idea has been introduced in Mousis et al. (2017b) but never investigated to quantitatively estimate the fraction of $\mathrm{O}_{2}$ produced during the vertical transport of grains. Due to turbulent mixing, the ice grains present in the midplane were lifted toward the upper layers of the disk and dragged down over a large number of cycles (Ciesla \& Sandford 2012; Mousis et al. 2017b). Consequently, these grains spent a non-negligible fraction of their lifetime in the disk's upper regions, where the irradiation by cosmic rays was strong. This irradiation, integrated over the disk lifetime, might cause some potentially significant production of $\mathrm{O}_{2}$, the extent of which is explored in the present work.

\section{Disk Model and Transport Module}

To mimic the vertical motion of particles, we used a simple description of the PSN structure (Chiang \& Goldreich 1997; Hartmann et al. 1998; Ciesla \& Sandford 2012). The gas surface density $\Sigma_{g}$, namely the gas column density integrated through the disk plane, and temperature $T_{d}$ profiles of the disk model are given by

$$
\Sigma_{g}(r)=2000\left(\frac{r}{1 \mathrm{au}}\right)^{-1} \mathrm{~g} \mathrm{~cm}^{-2}
$$




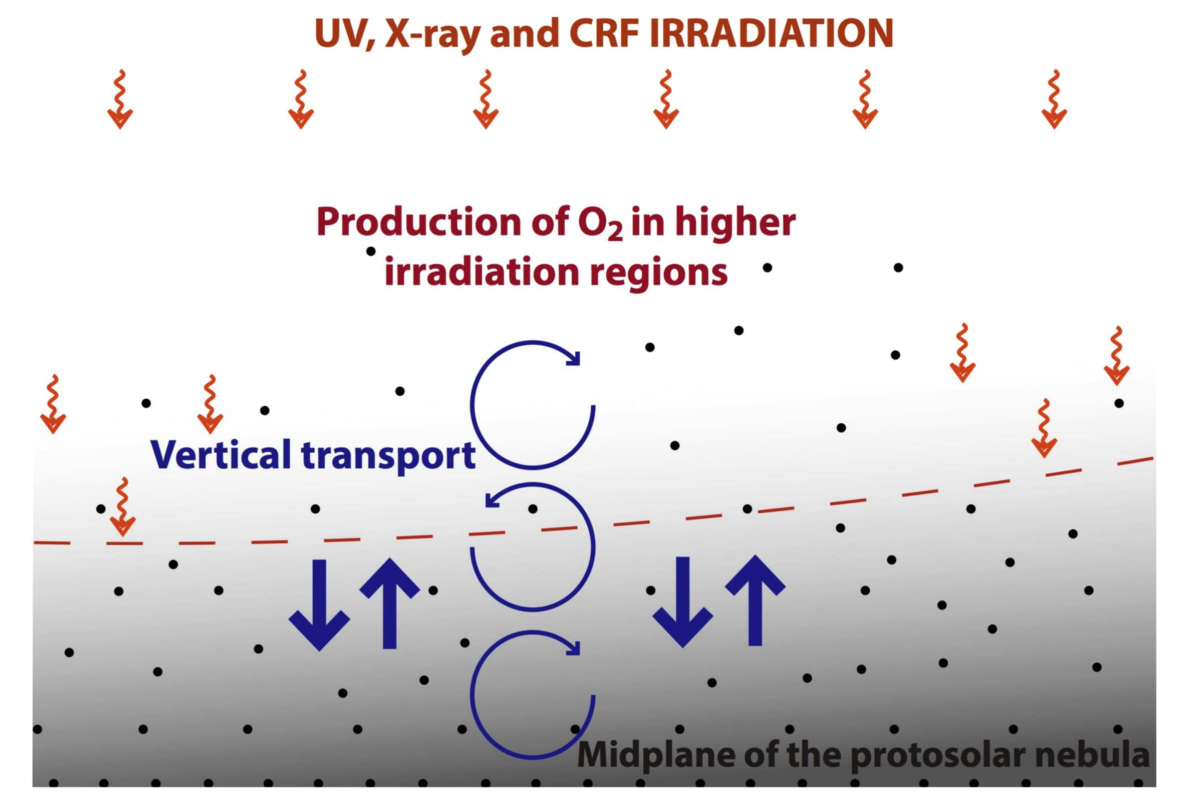

Figure 1. Illustration of the vertical transport of small icy grains toward disk regions where irradiation is strong and favors the formation of $\mathrm{O}_{2}$. Grains remain concentrated in the midplane of the disk because of gravitational settling and gas drag.

and

$$
T_{d}(r)=280\left(\frac{r}{1 \mathrm{au}}\right)^{-1 / 2} \mathrm{~K}
$$

where $r$ is the heliocentric distance. These prescriptions are appropriate for flared disks around young $\mathrm{T}$ Tauri stars.

Using the assumption of vertical hydrostatic equilibrium for the gas, the disk's density is expressed as a function of heliocentric distance $r$ and altitude $z$ above the midplane as

$$
\rho_{g}(r, z)=\rho_{0}(r) e^{-\frac{z^{2}}{2 H_{s}^{2}}},
$$

with

$$
\rho_{0}(r)=\frac{\Sigma_{g}(r)}{\sqrt{2 \pi} H_{g}},
$$

where $H_{g}=c_{g} / \Omega_{K}$ is the gas scale height derived from the isothermal gas sound speed $c_{g}=\sqrt{R_{g} T_{d} / \mu_{g}}$ and the keplerian orbital frequency $\Omega_{K}$. $R_{g}$ is the ideal gas constant and $\mu_{g}$ is the mean molecular weight of the gas $\left(\sim 2.4 \mathrm{~g} \mathrm{~mol}^{-1}\right)$.

Below we briefly outline the main aspects of the transport model used in our computations. We refer the reader to the work of Ronnet et al. (2017) for a full description. We consider the diffusion of dust grains onto the background disk gas using a Lagrangian approach where individual grains are tracked. Because our study only addresses the influence of irradiation during the vertical transport of grains, we opted not to follow their radial evolution. Small dust grains are strongly coupled and have a radial velocity similar to that of the background gas (and follow the accretion flow onto the star), whereas larger, partially decoupled grains, rapidly drift inward due to gas drag (e.g., Weidenschilling 1977). Considering the vertical hydrostatic equilibrium of the gas disk, we assume the gas has no net vertical velocity (see Takeuchi \& Lin 2002 for a discussion of this assumption).

The vertical motion of the grains is therefore ruled by the turbulent diffusion that lifts them toward the upper layers of the disk and the settling toward the midplane due to gas drag and the gravity of the central star. The latter is given by the equation of motion of the dust grains in the vertical direction, which is solved following the approach developed in Ronnet et al. (2017):

$$
\frac{d v_{d, z}}{d t}=-\frac{G M z}{r^{3}}-\frac{v_{d, z}}{t_{s}},
$$

where $v_{d, z}$ is the dust vertical velocity, $M=1 M_{\odot}$ is the mass of the central star, $z$ is the vertical position of the dust grain, and $t_{s}$ is the stopping time. The stopping time is a measure of the timescale on which the gas transfers its angular momentum to dust and can be expressed as (Perets \& Murray-Clay 2011)

$$
t_{s}=\left(\frac{\rho_{g} v_{\text {th }}}{\rho_{s} R_{s}} \min \left[1, \frac{3}{8} \frac{v_{\text {rel }}}{v_{\text {th }}} C_{D}(R e)\right]\right)^{-1},
$$

where $v_{\text {th }}=\sqrt{8 / \pi} c_{g}$ is the gas thermal velocity, $R_{s}$ is the radius of the solid particle, $\rho_{s}$ is its density, assumed to be $1 \mathrm{~g} \mathrm{~cm}^{-3}$ regardless of the size, and $v_{\text {rel }}$ is the relative velocity between the gas and the dust grain. The dimensionless drag coefficient $C_{D}$ is a function of the Reynolds number $R e$ of the flow around the particle and derives from an empirical law fitted on recent experimental data (Perets \& Murray-Clay 2011):

$$
C_{D}=\frac{24}{R e}(1+0.27 R e)^{0.43}+0.47\left(1-e^{-0.04 R e^{0.38}}\right),
$$

where $R e$ is the Reynolds number. It is given by (Supulver \& Lin 2000)

$$
R e=\frac{4 R_{s} v_{\mathrm{rel}}}{c_{g} l_{g}},
$$

where $l_{g}$ is the mean-free path of the gas.

The turbulent diffusion of the grains is modeled using a Monte-Carlo scheme where individual particles are given random impulses to mimic the stochastic transport due to turbulent eddies (see, e.g., Ciesla 2010, 2011). Overall, the new 
position $z_{d}$ of a dust grain after a timestep $d t$ is computed as follows in the vertical direction:

$$
z_{d}(t+d t)=z_{d}(t)+v_{\mathrm{adv}} d t+R_{1}\left[\frac{2}{\sigma^{2}} D_{p} d t\right]^{\frac{1}{2}}
$$

where $R_{1} \in[-1 ; 1]$ is a random number, $\sigma^{2}$ is the variance of the random number distribution, $D_{p}$ is the diffusivity of the solid particle, and $v_{\mathrm{adv}}$ is the term accounting for the nonuniform density of the gas in which the particles diffuse as well as the nonuniform diffusivity of the particles, and the forces experienced by the particle (see Equation (11)). The diffusivity of the dust grains $D_{p}$ is related to the gas diffusivity through the Schmidt number Sc as (Youdin \& Lithwick 2007):

$$
S c \equiv \frac{\nu}{D_{p}} \sim 1+S t^{2}
$$

where $\nu=\alpha H_{g}^{2} \Omega_{K}$ is the turbulent viscosity of the gas (assumed to be equivalent to the gas diffusivity) expressed through the nondimensional $\alpha$ parameter measuring the level of turbulence within the disk (Shakura \& Sunyaev 1973) and St is the Stokes number, corresponding to the stopping time multiplied by the local Keplerian frequency. Larger values of $\alpha$ yield a more efficient redistribution of the dust grains through turbulent diffusion. Finally, the advection term in the transport equation is given by Ciesla $(2010,2011)$ as

$$
v_{\mathrm{adv}}=\frac{D_{p}}{\rho_{g}} \frac{\partial \rho_{g}}{\partial z}+\frac{\partial D_{p}}{\partial z}+v_{d, z} .
$$

This set of equations allows us to derive the vertical position $z_{d}$ of individual particles at each timestep and to subsequently estimate the dose of irradiation they received.

\section{Irradiation of Grains}

The energy received by water molecules per unit time due to cosmic-ray irradiation $W_{\text {irr }}(n)$ as a function of the column density of gas $n$ above the particle is taken from Yeghikyan (2011). These authors computed this term by using the cosmicray intensity $I(E)\left(\mathrm{cm}^{-2} \mathrm{~s}^{-1} \mathrm{st}^{-1} \mathrm{MeV}^{-1}\right)$ from Cooper et al. (2003) and considering a planar geometry. This leads to a differential flux spectrum inside the disk $F(E)$ given by

$$
F(E)=\pi I(E)\left(\mathrm{cm}^{-2} \mathrm{~s}^{-1} \mathrm{MeV}^{-1}\right)
$$

$W_{\text {irr }}(n)$ is then derived from the molecular cloud material stopping power $S(E)$, i.e., its capability to absorb the cosmicray energy, as estimated from the Stopping and Range of Ions in Matter program (Ziegler et al. 2010). Note that the stopping power mostly depends on the mass density and not on the intrinsic composition or structure of the material. The computation is made for the proton component of the cosmic rays and the contribution of the alpha particles is estimated approximately, while the contribution of heavier cosmic-ray particles is neglected. We consider that the approach of Yeghikyan (2011) provides the best prescription of $W_{\text {irr }}(n)$ in the literature because (i) this term weakly depends on the composition, (ii) the planar hypothesis is consistent with the geometry of the disk, and (iii) the CRF derived from Cooper et al. (2003) is the best proxy available for the one received by the PSN.

At each timestep, the column density of gas above a given particle $p$ is calculated as

$$
n=\frac{N_{\mathrm{A}}}{\mu_{g}} \int_{\left|Z_{p}\right|}^{+\infty} \rho_{g}(z) d z,
$$

where $N_{\mathrm{A}}$ is Avogadro's number. The energy deposited onto water molecules during the timestep $d t$ is then given by

$$
E_{\text {dep }}=W_{\text {irr }}(n) d t .
$$

$\mathrm{O}_{2}$ is produced by radiolysis of water ice through the chemical reaction $2 \mathrm{H}_{2} \mathrm{O} \rightarrow 2 \mathrm{H}_{2}+\mathrm{O}_{2}$, with an amount of energy $E_{w}$ needed to alter one $\mathrm{H}_{2} \mathrm{O}$ molecule being $E_{w}=$ $235 \mathrm{eV}$ (Johnson 1991). The fraction of $\mathrm{O}_{2}$ produced by the alteration of two water molecules, assuming a full efficiency, is then

$$
\frac{\left[\mathrm{O}_{2}\right]}{\left[\mathrm{H}_{2} \mathrm{O}\right]}=\frac{E_{\mathrm{dep}}(t)}{2 E_{W}}
$$

Note that the value of $E_{w}=235 \mathrm{eV}$ is a lower limit, and that the "full efficiency" assumption may be too generous. In fact, Teolis et al. (2017) find for highly penetrating radiation (such as cosmic rays penetrating through grains) that average $\mathrm{G}$-values for $\mathrm{O}_{2}$ have an approximate inverse dependence on particle penetration range (see their Figure 2).

\section{Results}

Turbulence plays an important role in the motion of gascoupled small particles. Here, micron-sized grains that initially settled in the midplane are entrained by turbulent eddies and diffuse both radially and vertically with an effective viscosity roughly equal to that of the gas (Ciesla \& Sandford 2012). Consequently, solid particles follow a Gaussian distribution in the vertical direction. The scale height of dust (corresponding to the standard deviation of the distribution) is a fraction of the gas scale height, this fraction being larger and possibly equal to the gas scale height $H_{g}$ in the cases of small grains and higher degrees of turbulence.

Figure 2 represents the vertical distribution of $10^{-6}, 10^{-4}$, and $10^{-2} \mathrm{~m}$ particles computed at a fixed distance of 30 au from the Sun with our disk model with vertical transport, assuming a coefficient of turbulent viscosity $\alpha=10^{-3}$. The vertical transport of 2000 particles is simulated in each case. The figure shows that vertical spreading is more important in the cases of $10^{-6}$ and $10^{-4} \mathrm{~m}$ particles and can reach up to $2-3$ gas scale heights. In contrast, because of their larger size, $10^{-2} \mathrm{~m}$ particles are much less affected by turbulence and do not spread more than $\sim 0.1$ scale heights above the midplane. Figure 3 displays the vertical evolution of a $10^{-6} \mathrm{~m}$ particle integrated over $1 \mathrm{Myr}$ at $30 \mathrm{au}$ in the PSN. It also shows that this particle spends a non-negligible amount of time in the regions above the disk midplane, where irradiation is more significant. $10^{-4} \mathrm{~m}$ particles display similar behaviors to a slightly lower extent while $10^{-2} \mathrm{~m}$ particles remain mostly close to the disk midplane.

Figure 4 shows the time evolution of the $\mathrm{O}_{2} / \mathrm{H}_{2} \mathrm{O}$ ratio in $10^{-6}, 10^{-4}$, and $10^{-2}$ grains irradiated along their vertical 

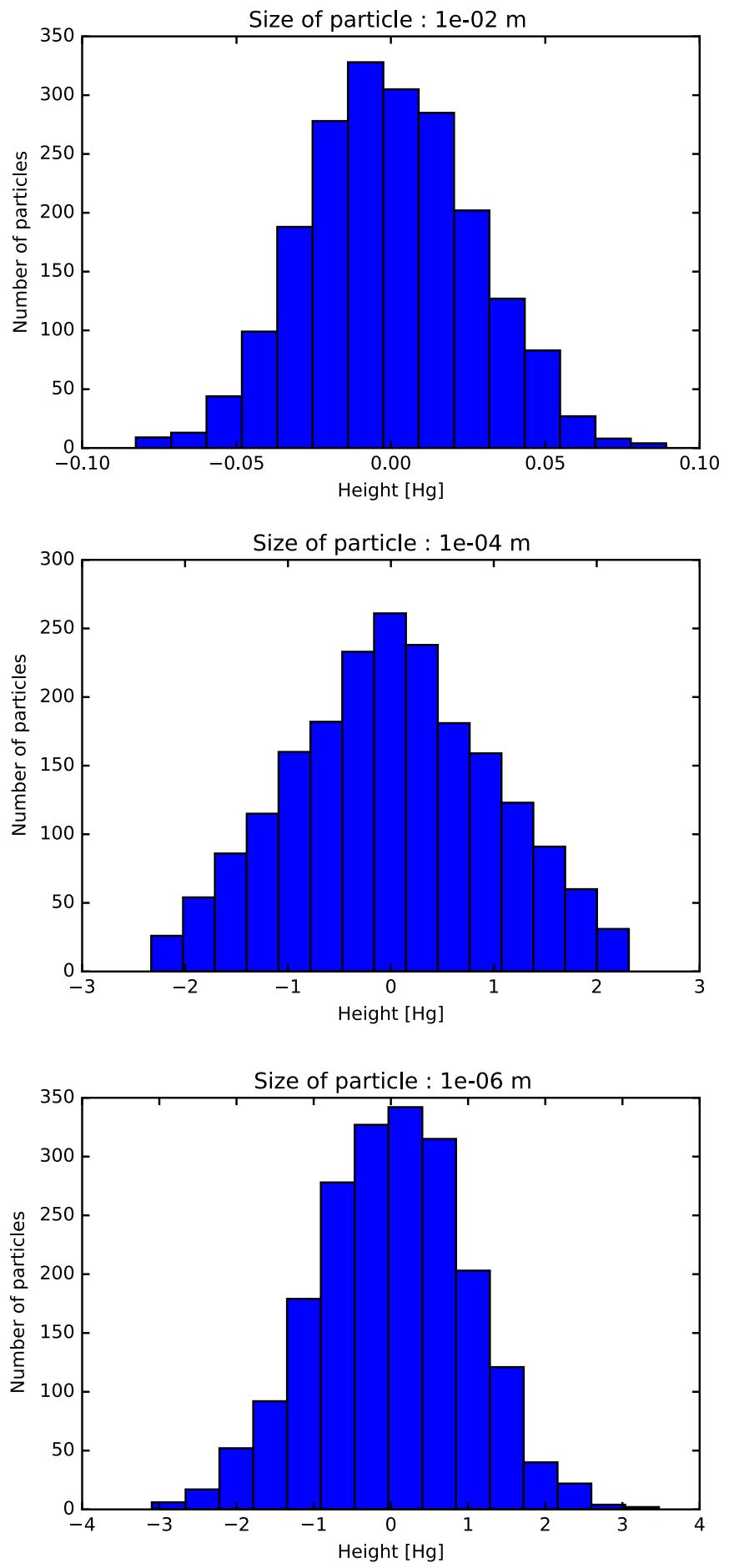

Figure 2. Vertical distribution of $10^{-6}, 10^{-4}$, and $10^{-2} \mathrm{~m}$ particles at 30 au in the PSN. The height above the disk is expressed in function of the disk's gas scale height $\left(H_{g}\right)$.

trajectories and for a turbulent viscosity $\alpha$ equal to $10^{-3}$ and $10^{-2}$, namely two typical values for the disk's viscosity parameter (Drouart et al. 1999). The resulting $\mathrm{O}_{2} / \mathrm{H}_{2} \mathrm{O}$ ratio in $10^{-6} \mathrm{~m}$ particles (the most favorable case) is at best $\sim 10^{-5}$, namely three orders of magnitude lower than the one observed in $67 \mathrm{P} / \mathrm{C}-\mathrm{G}$ by the Rosetta spacecraft, after $1 \mathrm{Myr}$ of vertical transport in the PSN, irrespective of the adopted $\alpha$ value. After

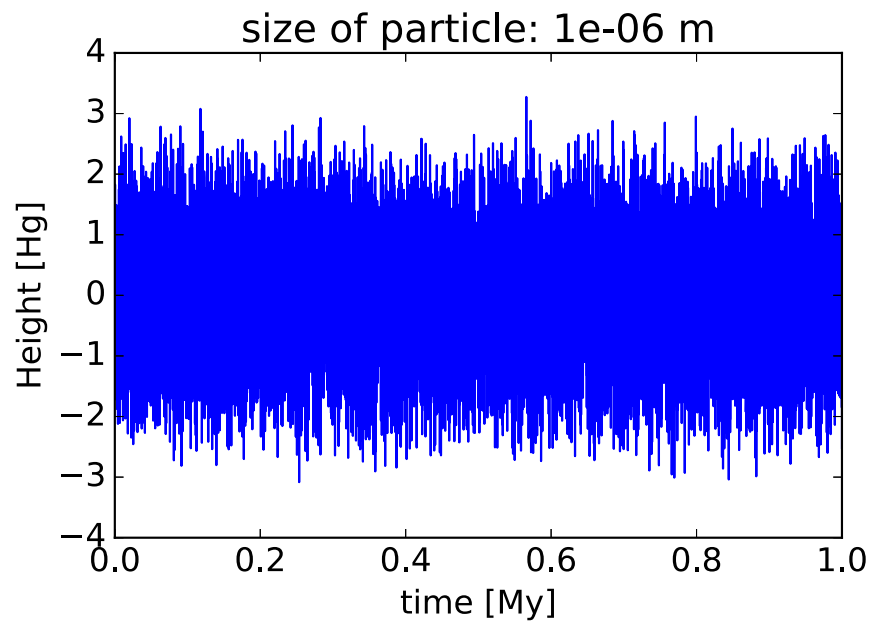

Figure 3. Vertical evolution of a $10^{-6} \mathrm{~m}$ particle as a function of time at $30 \mathrm{au}$ in the PSN.
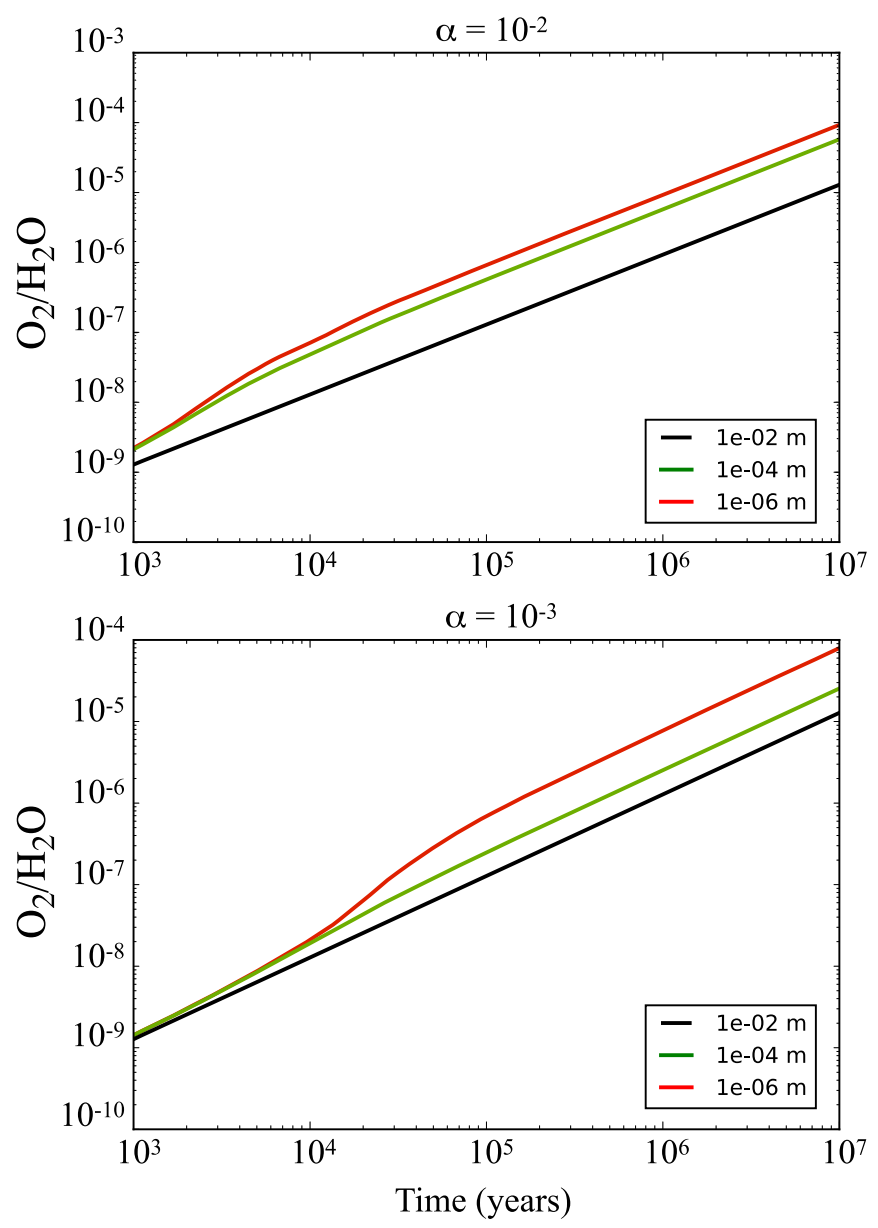

Figure 4. Abundance of $\mathrm{O}_{2}$ relative to $\mathrm{H}_{2} \mathrm{O}$ in $10^{-6}, 10^{-4}$, and $10^{-2} \mathrm{~m}$ particles as a function of time in the PSN and for $\alpha$ values equal to $10^{-2}$ (top panel) and $10^{-3}$ (bottom panel).

the same timespan, the $\mathrm{O}_{2} / \mathrm{H}_{2} \mathrm{O}$ ratio reaches no more than $\sim 10^{-6}$ in $10^{-2} \mathrm{~m}$ particles, while the one obtained in $10^{-4} \mathrm{~m}$ grains is in the $\sim 2-6 \times 10^{-6}$ range, depending on the chosen 
value of $\alpha$. After $10 \mathrm{Myr}$ of vertical transport, the $\mathrm{O}_{2} / \mathrm{H}_{2} \mathrm{O}$ ratio only increases by one order of magnitude in each considered case, leading to a $\mathrm{O}_{2} / \mathrm{H}_{2} \mathrm{O}$ ratio of $\sim 10^{-4}$ in the most favorable situation $\left(10^{-6} \mathrm{~m}\right.$ particles), which is still two orders of magnitude lower than the value observed in $67 \mathrm{P} / \mathrm{C}-\mathrm{G}$.

\section{Discussion}

Our computations suggest that, even if a significant fraction of the icy particles has followed a back and forth cycle toward the upper layers of the disk over tens of millions of years in a static PSN, the amount of $\mathrm{O}_{2}$ created via radiolysis is at least $\sim$ two orders of magnitude lower than the Rosetta observations. This timespan exceeds by far the formation timescale of $67 \mathrm{P} / \mathrm{C}-\mathrm{G}$, which has been estimated to range between 2.2 and 7.7 Myr after the formation of $\mathrm{Ca}-\mathrm{Al}$-rich inclusions in the PSN (Mousis et al. 2017a). The $\mathrm{O}_{2} / \mathrm{H}_{2} \mathrm{O}$ ratios derived from our simulations at $1 \mathrm{Myr}$ can already be considered as optimistic since the particles most likely grew and decoupled from gas after a few dozens of thousands of years of the disk evolution (Weidenschilling \& Cuzzi 1993). In this case, once the icy grains have grown up to sizes larger than a few meters in the PSN, the bulk of the ice should remain unaltered by irradiation. Also, an increase of the CRF up to a factor of $\sim 100$ due to a close supernova explosion would not substantially change the $\mathrm{O}_{2} / \mathrm{H}_{2} \mathrm{O}$ ratio in icy grains because the timespan of such an event (a few kyr) is too short (Mousis et al. 2016).

Several alternative mechanisms have recently been investigated in the literature to account for the $\mathrm{O}_{2}$ detection in $67 \mathrm{P} / \mathrm{C}-\mathrm{G}$. Among them, Taquet et al. (2016) have proposed that $\mathrm{O}_{2}$ could be produced in dark clouds via a combination of gas-phase and solidstate chemical reactions leading to its formation and destruction, in agreement with the ROSINA observations and the conclusions of Mousis et al. (2016). Another mechanism, proposed by Dulieu et al. (2017), consists of the production of $\mathrm{O}_{2}$ through dismutation of $\mathrm{H}_{2} \mathrm{O}_{2}$ during water ice desorption from the nucleus. However, this mechanism requires the incorporation of large amounts of primordial $\mathrm{H}_{2} \mathrm{O}_{2}$ into the nucleus and its complete conversion into $\mathrm{O}_{2}$ to be consistent with the low levels of $\mathrm{H}_{2} \mathrm{O}_{2}$ observed in the coma. Another scenario investigated by Yao \& Giapis (2017) is the possible present-day production of $\mathrm{O}_{2}$ via an Eley-Rideal reaction mechanism in the coma. This reaction between energetic water ions and adsorbed O-atoms, produces highly excited oxywater $\left(\mathrm{H}_{2} \mathrm{O}_{2}\right)$, which undergoes delayed fragmentation to form $\mathrm{HO}_{2}$ as the precursor for $\mathrm{O}_{2}$. However, at a close distance to the Sun, the solar wind strengthens and increases the ionization and water ion flux to the surface of the nucleus, and consequently produces more $\mathrm{O}_{2}$ if the Eley-Rideal reaction mechanism is effective. According to this mechanism, the $\mathrm{O}_{2} / \mathrm{H}_{2} \mathrm{O}$ ratio should increase at perihelion, a trend that has not been seen by the ROSINA instrument.

Our results thus favor the mechanism of radiolysis of icy grains in low-density environments such as the presolar cloud, shown by Mousis et al. (2016) to be capable of producing the $\mathrm{O}_{2}$ abundance observed in $67 \mathrm{P} / \mathrm{C}-\mathrm{G}$. They suggested that $\mathrm{O}_{2}$ may be trapped in the grains in radiation defects/cavities, and subsequently delivered to the PSN either in the solid or gas phase, depending on the disk's thermodynamic structure. The constant $\mathrm{O}_{2} / \mathrm{H}_{2} \mathrm{O}$ ratio observed in $67 \mathrm{P} / \mathrm{C}-\mathrm{G}$ requires that both $\mathrm{O}_{2}$ and $\mathrm{H}_{2} \mathrm{O}$ were released from the same solid phase and supports the hypothesis suggesting that comets formed from water ice coming from ISM without suffering from vaporization when entering the PSN. ${ }^{7}$ Relaxing this constraint would leave alternative delivery scenarios of $\mathrm{O}_{2}$ to comets, among which the desorption of this molecule during the amorphous-to-crystalline ice phase transition was encompassed by presolar grains that entered into the disk. The $\mathrm{O}_{2}$ released via this manner could have been subsequently trapped in clathrates during the cooling of the PSN before being incorporated in the building blocks of comets. Finally, we note that, in addition to $\mathrm{O}_{2}$ formation in low-density environments at epochs prior to the genesis of the protosolar nebula, an endogenic radiolytic source due to radionuclides present in the dusty component of the comet nucleus may have contributed at the percent level to the total $\mathrm{O}_{2}$ budget in comets (Bouquet et al. 2017).

We thank the anonymous referee for helpful comments. O.M. acknowledges support from CNES. O.M. and T.R. acknowledge support from the $A^{*}$ MIDEX project ( $n^{\circ}$ ANR-11IDEX-0001-02) funded by the "Investissements d'Avenir" French Government program, managed by the French National Research Agency (ANR). J.I.L. was supported by the JWST project through a grant from NASA/GSFC.

\section{ORCID iDs}

R. Maggiolo (iD https://orcid.org/0000-0002-5658-1313

P. Wurz (iD https://orcid.org/0000-0002-2603-1169

A. Bouquet (iD https://orcid.org/0000-0001-8262-9678

\section{References}

Bieler, A., Altwegg, K., Balsiger, H., et al. 2015, Natur, 526, 678 Bouquet, A., Mousis, O., Teolis, B., et al. 2017, ApJL, submitted Chiang, E. I., \& Goldreich, P. 1997, ApJ, 490, 368

Ciesla, F. J. 2010, ApJ, 723, 514

Ciesla, F. J. 2011, ApJ, 740, 9

Ciesla, F. J., \& Sandford, S. A. 2012, Sci, 336, 452

Cooper, J. F., Christian, E. R., Richardson, J. D., \& Wang, C. 2003, EM\&P, 92, 261

Drouart, A., Dubrulle, B., Gautier, D., \& Robert, F. 1999, Icar, 140, 129

Dulieu, F., Minissale, M., \& Bockelée-Morvan, D. 2017, A\&A, 597, A56

Hartmann, L., Calvet, N., Gullbring, E., \& D’Alessio, P. 1998, ApJ, 495, 385

Johnson, R. E. 1991, JGR, 96, 17

Mousis, O., Drouard, A., Vernazza, P., et al. 2017a, ApJL, 839, L4

Mousis, O., Ozgurel, O., Lunine, J. I., et al. 2017b, ApJ, 835, 134

Mousis, O., Ronnet, T., Brugger, B., et al. 2016, ApJL, 823, L41

Perets, H. B., \& Murray-Clay, R. A. 2011, ApJ, 733, 56

Ronnet, T., Mousis, O., \& Vernazza, P. 2017, ApJ, 845, 92

Rubin, M., Altwegg, K., van Dishoeck, E. F., \& Schwehm, G. 2015, ApJL, 815, L11

Shakura, N. I., \& Sunyaev, R. A. 1973, A\&A, 24, 337

Supulver, K. D., \& Lin, D. N. C. 2000, Icar, 146, 525

Takeuchi, T., \& Lin, D. N. C. 2002, ApJ, 581, 1344

Taquet, V., Furuya, K., Walsh, C., \& van Dishoeck, E. F. 2016, MNRAS, 462, S99

Teolis, B. D., Plainaki, C., Cassidy, T. A., \& Raut, U. 2017, JGRE, 122, 1996 Weidenschilling, S. J. 1977, MNRAS, 180, 57

Weidenschilling, S. J., \& Cuzzi, J. N. 1993, in Protostars and Planets III, ed.

E. H. Levy \& J. I. Lunine (Tucson, AZ: Univ. Arizona Press), 1031

Yao, Y., \& Giapis, K. P. 2017, NatCo, 8, 15298

Yeghikyan, A. G. 2011, Ap, 54, 87

Youdin, A. N., \& Lithwick, Y. 2007, Icar, 192, 588

Ziegler, J. F., Ziegler, M. D., \& Biersack, J. P. 2010, NIMPB, 268, 1818

\footnotetext{
This scenario does not preclude a possible amorphous-to-crystalline ice phase transition due to a moderate PSN temperature along the migration path of the grains (Mousis et al. 2016).
} 